\title{
Die Blockfreienbewegung im Streit um die Malwinen (Falklands)
}

von Klaus Fritsche

Uber ein Jahr ist vergangen, seit sich Großbritannien und Argentinien eine blutige Schlacht um die Malwinen (Falklands) lieferten. ${ }^{1}$ Wer aber hoffte, daß nach der Feuereinstellung zügig die Verhandlungswege zu einer friedlichen Lösung begangen würden, sah sich enttäuscht. Die Fronten sind verhärtet wie lange nicht mehr. Eine Konfliktlösung ist nicht in Sicht. Großbritannien weigert sich sogar, überhaupt Verhandlungen über die Souveränitätsfrage zu führen² und zeigt mit demonstrativen Gesten Flagge, wie mit dem Blitzbesuch von Premierministerin Thatcher im Januar 1983.

Der Krieg um die Malwinen (Falklands) berührte aber nicht nur die zweiseitigen Beziehungen zwischen Argentinien und Großbritannien, sondern hatte weitreichende Folgen für die gesamte außenpolitische Orientierung Lateinamerikas. Unübersehbar ist die eingetretene "Entfremdung" des Kontinents von den USA, verbunden mit einer stärkeren Hinwendung dieser Länder, einschließlich der westlich orientierten, zur Blockfreienbewegung.

Diese Entwicklung ist sicherlich nicht nur eine Antwort auf die probritische Haltung der USA im Malwinen-Konflikt, sondern auch ein Ergebnis des Verhaltens der Blockfreien in dieser Frage. Angesichts der Bedeutung dieser Entwicklung Grund genug, die Frage zu untersuchen, welche Position die Blockfreienbewegung in diesem Streitfall eingenommen und welche Vorschläge zur Konfliktlösung sie unterbreitet haben, zumal mit Argentinien ein Mitglied der Blockfreienbewegung selbst diesen Krieg begonnen hat. ${ }^{3}$

\section{Die Malwinen-Frage auf den Blockfreienkonferenzen bis 1981}

Die Blockfreienbewegung befaßte sich zum ersten Mal auf ihrer Außenministerkonferenz in Lima (August 1975) mit dieser Frage. Im Paragraphen 87 der Schlußdeklaration heißt es:

1 Zur Chronik und Dokumentation des Konflikts siehe: Dokumente zum Konflikt um die Falklandinseln, in: Europa-Archiv, 37. Jg., 19. Folge, D 473 - D 508; eine Aufstellung aller im Rahmen der UN zirkulierten Dokumente während des Konflikts in UN-Document A/AC.109/712/Addl; Diskussion über die völkerrechtliche Einschätzung in: Hermann Weber, "Falkland-Islands" oder "Malvinas"?, Frankfurt 1977, wie die Diskussion zwischen H. Weber und Rudolf Dolzer in der FAZ vom 5. 4. und 29. 4. 1982; zur sowjetischen Position siehe: Die sowjetische Presse zum Falkland-Konflikt, in: Osteuropa 11/1982, A 608-A 619.

2 Vgl. dazu die Stellungnahme Großbritanniens anläßlich der UN-Debatte im November 1982, sowie die wiederholten Erklärungen der britischen Premierministerin zu dieser Frage im Januar 1983.

3 Argentinien wurde 1973 Vollmitglied der Blockfreienbewegung, nachdem es seit 1964 als Beobachter an den Gipfelkonferenzen der Blockfreienbewegung teilgenommen hatte. Hier wird die Entwicklung bis Nov. 1982 untersucht. 
"Die Blockfreien Länder, unbeschadet der Bestätigung der Gültigkeit des Prinzips der Selbstbestimmung als allgemeingültiges Prinzip für die übrigen Gebiete, unterstützen in diesem besonderen einzelnen Fall der Malwinen den gerechten Anspruch der Republik Argentinien und fordern Großbritannien auf, aktiv die von den Vereinten Nationen empfohlenen Verhandlungen fortzusetzen, damit diese Inselgruppe wieder unter die argentinische Souveränität kommt und somit diesem illegalen Zustand ein Ende bereitet wird, der immer noch im südlichen Teil des amerikanischen Kontinents herrscht. $"{ }^{4}$

Diese Unterstützung des argentinischen Souveränitätsanspruchs, der auf friedlichem Wege realisiert werden soll, konnte auf die Stellungnahmen der Vereinten Nationen Bezug nehmen. Dort wurde die Malwinen-Frage unzweideutig als Problem der Dekolonisierung betrachtet. Bereits 1946 nahm die Generalversammlung die Inseln in die Liste der Gebiete ohne Selbstregierung auf. "Nach der Annahme der Dekolonisierungsresolution 1514 (XV) im Jahre 1960 wurden die Falklandinseln in der ,Vorläufigen Liste der Gebiete, auf die die Resolution GA 1514 (XV) anwendbar ist', geführt, und zwar in der Gruppe der 'Gebiete ohne Selbstregierung, für die von der Verwaltungsmacht Mitteilungen erfolgen'. Seit Oktober 1976 werden die Falklandinseln in der Liste der 'Gebiete, die bislang keine Unabhängigkeit erlangt haben', geführt. “"

Als Kolonialproblem wurde die Malwinen-Frage auch behandelt, als sich Argentinien 1964 mit dem Streit um die Inseln an die Vereinten Nationen wandte. ${ }^{6}$ In der am 16. Dezember 1965 verabschiedeten Resolution 2065 (XX), ${ }^{7}$ wird explizit das Ziel formuliert, "den Kolonialismus in jeder Form, auch wie er im Fall der Falklandinseln (Malwinen) in Erscheinung tritt, überall zu beenden." Argentiniens Anspruch auf die Inseln wird jedoch nicht anerkannt. Wie in der acht Jahre später verabschiedeten Resolution 3160 $(\mathrm{XXVIII})^{8}$ beschränkt man sich darauf, Argentinien und England zu einer friedlichen Lösung des Problems zu drängen, wobei "die Bestimmungen und Ziele der Charta der Vereinten Nationen und der Resolution 1514 (XV) sowie die Interessen der Bewohner der Falklandinseln (Malwinen) zu berücksichtigen sind ". ${ }^{9}$

In der Diskussion über die Frage, ob dem Selbstbestimmungsrecht der Falkländer, die ja von britischen Siedlern abstammen, oder der territorialen Einheit Argentiniens Vorrang eingeräumt werden soll, nehmen diese Resolutionen nur indirekt Stellung. Sie räumen den Falkländern kein explizites Selbstbestimmungsrecht ein, behandeln die Frage als ei-

4 Ubersetzt nach UN-Document S/15003, S. 2. Die Úbersetzung in der Internationalen Politik (Belgrad), No. 611, S. 29 ist sinnentstellend.

5 Hermann Weber, a.a.O., S. 40 f. (1).

6 Zuständig ist der sog. 25er-"Sonderausschuß für den Stand der Verwirklichung der Erklärung über die Gewährung der Unabhängigkeit an koloniale Länder und Völker“. Zur Mitgliedschaft im Jahr 1982 s. Vereinte Nationen, Heft 5/1982, S. 184.

7 In: Vereinte Nationen, Bonn, Heft 3/1982, S. $106 \mathrm{f}$

8 Ebenda, S. 107.

9 RES 2065 (XX). 
nen Souveränitätskonflikt zwischen Argentinien und Großbritannien und fordern nur, "die Interessen der Bewohner . . . zu berücksichtigen" ". ${ }^{10}$

Die Blockfreien haben seit der Außenministerkonferenz von Lima klar gegen das "Selbstbestimmungsrecht der Falkländer" Stellung bezogen und die Malwinen als "besonderen einzelnen Fall« erklärt. Den Grund dafür erläuterte noch einmal der mexikanische Vertreter Munoz Ledo am 3. November 1982 vor der 37: Generalverammlung der Vereinten Nationen.

"Die Generalversammlung hat beständig ihre Position bekräftigt, in diesem Fall nicht das Selbstbestimmungsrecht anzuwenden, sich die Differenz zwischen echten Kolonien und territorialen Enklaven vor Augen führend . . . Wer kann den Anspruch erheben, daß die Einwohner von territorialen, strategischen und militärischen Enklaven der Großmächte, die Bürger derselben Großmächte sind, das Recht auf Selbstbestimmung auf dem Territorium eines anderen Staates in Anspruch nehmen können? Wer könnte damit übereinstimmen, daß die südafrikanischen Bewohner der Walfisch Bay ... das Recht auf Selbstbestimmung ausüben würden und dieses Gebiet vom Territorium Namibias abtrennen würden, oder das die britische Bevölkerung von Gibraltar sich als Karrikatur einer neuen Nation auf spanischem Territorium aufspielen könnte. "11

Sicherlich nicht zufällig kam nach dem Beschluß der Blockfreienbewegung von 1975, der auf der 5. Gipfelkonferenz Blockfreier Staaten in Colombo 1976 bekräftigt wurde ${ }^{12}$ erneut Bewegung in die Sache, und die Malwinen-Frage vor die 31. Generalversammlung 1976. Die dort verabschiedete Resolution $31 / 49^{13}$ nahm eindeutiger zugunsten Argentiniens Stellung. In der Präambel wird auf die Beschlüsse der Blockfreienkonferenzen hingewiesen. Argentinien wird gedankt "für die . . . ständigen Bemühungen, den Entkolonisierungsprozeß zu erleichtern und das Wohlergehen der Bevölkerung der Inseln zu fördern". Ein gesonderter Paragraph, nach dem die Interessen der Bewohner der Malwinen bei der Lösung zu berücksichtigen sind, wurde nicht mehr aufgenommen.

Entsprechend war auch das Abstimmungsverhalten. Großbritannien, das sich bei den vorherigen Abstimmungen der Stimme enthalten hatte, stimmte gegen diese Resolution. Der britische UN-Vertreter Dalton begründete diese Haltung wie folgt: Die Resolution 31/49 ginge weiter als die vorhergegangenen Resolutionen. "Sie erwähnt nicht das Prinzip der Selbstbestimmung. Man hat uns gesagt, daß die Falklands ein spezieller Fall seien. Wir können das nicht akzeptieren." Deshalb werden wir "gegen den Resolutionsentwurf stimmen . .., weil wir nicht akzeptieren können, daß das einzige Ergebnis solcher Kontakte (mit Argentinien, KF) das Ubertragen der Souveränität auf Argentinien sein kann . . ."14

10 Ebenda.

11 A/37/PV.52, 3. 11. 1982.

12 Punkt 119 der Schlußdeklaration der 5. Gipfelkonferenz von Colombo, in: Die Bewegung der Blockfreien, Archiv Dritte Welt, Köln 1978, S. 189.

13 In Vereinte Nationen, Bonn, Heft 4/1982, S. 147 f.

14 Official Records of the General Assembly, Thirty First Session, 85th Meeting, S. 1293, Punkt 25 und 26. 
Auch die Zahl der Enthaltungen stieg bei dieser Abstimmung stark an. Bei den Resolutionen von 1965 und 1973 gab es keine Gegenstimmen, aber jeweils 14 Enthaltungen, die - mit Ausnahme Südafrikas - aus den westlichen Industrieländern kamen. 1976 stieg die Zahl der Enthaltungen auf 32. Diesmal auch mit Gambia, Guayana, Jamaica, Kenia, Malawi, Sierra Leone, Singapur, Trinidad und Tobago und Zaire neun Mitglieder der Blockfreienbewegung. ${ }^{15}$

In den folgenden Jahren bestätigte die Blockfreienbewegung ihre Position und forderte seit 1978 immer wieder eine Beschleunigung der Verhandlungen. ${ }^{16}$ 1981, auf dem Treffen der Außenminister und Delegationsleiter anläßlich der 36sten Generalversammlung der Vereinten Nationen am 25. und 28. September, wurde erneut erklärt:

"Das Treffen bekräftigt seine Unterstützung der Rechte Argentiniens auf die Rückerstattung der Malwinen und die territoriale Souveränität über die Inseln und fordert, daß die diesbezüglichen Verhandlungen mit dem Vereinigten Königreich beschleunigt werden sollen. «"17

Welche Haltung nahmen nun die Blockfreien nach Ausbruch der offenen Feindseligkeiten ein. Obwohl sich die afrikanischen und asiatischen Blockfreien - anders als die Lateinamerikaner - mit öffentlichen Stellungnahmen zurückhielten, ${ }^{17 \mathrm{a}}$ kann ihre Reaktion anhand des Verhaltens der blockfreien Mitglieder im Sicherheitsrat, wie anhand der Stellungnahmen des Koordinationsbüros dieser Bewegung geprüft werden, das sich am 6. April, 26. April und am 5. Mai in New York auf Botschafterebene, und vom 31. Mai bis zum 5. Juni in Havanna auf Ministerebene mit dieser Frage befaßte.

\section{Die Reaktion auf die argentinische Invasion der Malwinen}

Schon die Diskussionen im Weltsicherheitsrat am 2./3. April machten Differenzen unter den blockfreien Mitgliedern deutlich. Der Resolution 502 (1982) ${ }^{18}$ stimmten außer Pana-

15 Enthaltungen bei der Resolution 2065 (XX): Australien, Dänemark, Finnland, Frankreich, Großbritannien, Island, Kanada, Neuseeland, Niederlande, Norwegen, Schweden, Portugal, Südafrika, USA.

Enthaltungen bei der Resolution 3160 (XXVIII): Belgien, Dänemark, BRD, Finnland, Frankreich, Großbritannien, Kanada, Luxemburg, Niederlande, Norwegen, Portugal, Schweden, Südafrika, USA. Die übrigen Enthaltungen bei der Resolution 31/49 kamen von Australien, Bahamas, Barbados, Belgien, Fidschi, Kanada, Dänemark, Finnland, Frankreich, BRD, Island, Irland, Italien, Japan, Luxemburg, Niederlande, Neuseeland, Norwegen, Papua-Neuguinea, Portugal, Schweden, USA, Osterreich.

Außer Zaire waren alle Mitglieder der Blockfreienbewegung, die sich der Stimme enthielten, gleichzeitig Mitglied des Commonwealth of Nations.

16 Entsprechende Passagen sind enthalten in den Schlußdeklarationen der Außenministerkonferenz in Belgrad 1979, Punkt 124, in: Internationale Politik (Belgrad), No. 680-1, S. 24; der 6. Gipf elkonferenz von Havanna 1979, Punkt 155), in: Internationale Politik (Belgrad), No. 707, S. 30; der Außenministerkonferenz von Neu Delhi 1981, Punkt 34 a, in: Internationale Politik, (Belgrad), No. 741, Dokumentation, S. 29.

17 UN-Document A/36/566 - S/14713, S. 18, Punkt X.

17a Zum Verhalten der ost- und südostasiatischen Länder vgl. Philip Bowring, Ripples from a storm, in: Far Eastern Economic Review, Nr. 26, 25. 6. 1982.

18 In: Vereinte Nationen, Bonn, Heft 3/1982, S. 106. 
ma die blockfreien Länder zu: Guayana, Jordanien, Togo, Uganda und Zaire. ${ }^{19}$ Sie unterstützten damit die Forderung nach "unverzüglichem Abzug aller argentinischen Streitkräfte" und nach einer diplomatischen Lösung, sowie die impliziet enthaltene Verurteilung der argentinischen Invasion.

Panama dagegen brachte darüberhinaus einen eigenen Resolutionsentwurf ein, ${ }^{20}$ der aber nicht zur Abstimmung gestellt wurde. Dieser enthält $u$. a. eine Unterstützung des argentinischen Souveränitätsanspruchs, verurteilt die Absichten Großbritanniens als Bedrohung des Weltfriedens und der internationalen Sicherheit und fordert Großbritannien auf, sich jeglicher Gewaltanwendung zu enthalten. Er wersucht beide Regierungen, unverzüglich Verhandlungen abzuhalten . . . und dabei sowohl die argentinische Souveränität . . . als auch die Interessen ihrer Bewohner gebührend zu achten." Panama brachte damit die mehrheitliche Meinung der lateinamerikanischen Staaten zum Ausdruck.

Demgegenüber wies der jordanische Vertreter - bei Unterstützung des argentinischen Anspruchs auf die Inseln - darauf hin, "daß die Blockfreienbewegung ihr moralisches Gesicht verlieren würde, wenn sie die universelle Charta «"11 der Vereinten Nationen verletzen würde. Uganda bedauerte "tief die Methoden, die die Republik Argentinien angewandt hat". Sie "bedauert die . . . Entwicklungen, weil sie sich der Tatsache bewußt ist, daß ähnliche, wenn auch nicht identische Streitfragen in anderen Teilen der Welt existieren. Es wäre ein gefährlicher Präzedenzfall, wenn der Eindruck entstehen würde, daß die Anwendung von Gewalt eine akzeptable Methode zur Lösung dieser Streitigkeiten sei". ${ }^{22}$

Das Koordinationsbüro der Blockfreien in New York befaßte sich in der ersten Phase der Auseinandersetzungen auf argentinischem Antrag zweimal mit der Malwinen-Frage. Nachdem auf der Sitzung vom 6. April keine Erklärung verabschiedet wurde, ${ }^{23}$ forderte das Büro am 26. April, die Unterstützung des argentinischen Anspruchs bestätigend, "eine gerechte, dauerhafte und friedliche Verhandlungslösung in Übereinstimmung mit der Anwendung der Sicherheitsrats-Resolution 502 (1982) in seiner Gesamtheit, den Prinzipien und Entscheidungen der Blockfreienbewegung und den entsprechenden Resolutionen der Generalversammlung". ${ }^{24}$ Es bekräftigt darüberhinaus "den Standpunkt, daß die Anwendung von Gewalt oder die Androhung von Gewaltanwendung in den Beziehungen zwischen Staaten den Prinzipien der Bewegung Blockfreier Staaten widerspricht".

Mit dieser Resolution wird der prinzipielle Standpunkt der Blockfreien bestätigt: Unterstützung des argentinischen Anspruchs auf die Inseln, Mißbilligung von Anwendung

19 Die weiteren Mitglieder des Sicherheitsrates waren USA, Großbritannien, Frankreich, China und die UdSSR als ständige Mitglieder; Irland, Japan, Polen und Spanien als nicht-ständige Mitglieder. China, Polen, UdSSR und Spanien enthielten sich der Stimme.

$20 \mathrm{~S} / 14950$, in deutsch als inoffizielle Vorauskopie.

21 United Nations, Security Council, Provisional Verbatim Record, S/PV. 2350, S. 26.

22 A.a.O., S. 91.

23 Entfällt.

$24 \mathrm{~S} / 15003$, S. 2., Hervorhebung durch KF. 
von Gewalt und damit impliziet, eine Verurteilung des argentinischen Vorgehens, das durch die Unterstützung der Resolution 502 (1982) verstärkt wird.

Gleichzeitig drückt die Mehrheit der Blockfreien aber die Hoffnung aus, daß nun andererseits England nicht seine bereitstehende Flotte zur Durchsetzung seiner anachronistischen und ungerechtfertigten Forderungen einsetzt und damit die Spannungen erhöht. Auch wenn die Forderung nach Anwendung der Resolution 502 (1982) in "ihrer Gesamtheit" in der Folgezeit auch unter den Blockfreien unterschiedlich interpretiert wird ${ }^{25}$ wird dies dadurch unterstrichen.

\section{Die Haltung nach dem Ausbruch offener Auseinandersetzungen}

Nach dem Scheitern der Vermittlungsbemühungen von US-Außenminister Haig und von Generalsekretär Perez de Cuellar, beginnt Großbritannien am 1. Mai mit der Bombardierung der Inseln und versenkt am 2. Mai außerhalb der 200-Meilen-"Ausschlußzone" den argentinischen Kreuzer "General Belgano". Am 3. Mai wird der Zerstörer "Sheffield" versenkt.

Unter dem Eindruck dieser Ereignisse tritt am 5. Mai das Koordinationsbüro erneut zusammen. In einem Kommuniqué ${ }^{26}$ wurde festgestellt, daß die "letzten Entwicklungen" die "Spannungen in der Region verstärkt haben und Frieden und Sicherheit in der Region wie in der ganzen Welt bedrohen". Gleichzeitig werden noch einmal "alle Aspekte" der Erklärung vom 26. April 1982 bekräftigt, insbesondere auch das Gebot der Nichtanwendung von Gewalt.

Am gleichen Tag betont der Präsident des Sicherheitsrates seine "Besorgnis über die Verschlimmerung der Lage in der Region" und unterstützt die "Bemühungen des Generalsekretärs", ${ }^{27}$ Kontakte zu den Konfliktparteien aufzunehmen. Das Ergebnis dieser Bemühungen diskutierte der Sicherheitsrat vom 21.-26. Mai 1982. Nach langer Diskussion, an der erneut eine große Zahl von Nichtmitgliedern des Sicherheitsrates beteiligten, wurde die Resolution 505 (1982) einstimmig verabschiedet. ${ }^{28}$ Diese fordert eine Einstellung der Feindseligkeiten, konzentriert sich aber auf die Unterstützung der Vermittlungsbemühungen des UN-Generalsekretärs.

In dieser Sicherheitsrats-Debatte wurde erneut die Spannbreite der Positionen der blockfreien Länder deutlich. Offensichtlich war ein tiefer Riß zwischen ihnen aufgetreten. Streitpunkt war die Haltung gegenüber der britischen Militäraktion. Während eine grö-

25 Die Auseinandersetzung über die Auslegung der Resolution 502 (1982) bezog sich auf folgende Frage. Besteht ein Junktim zwischen Abzug der Argentinier und Einstellung der Feindseligkeiten oder nicht. Diese Interpretation ging davon aus, daß Großbritannien nicht berechtigt war, selbst zu militärischen Aktionen zu greifen. Demgegenüber interpretierte England einen Zusammenhang zwischen der Feststellung der Resolution, daß ein Friedensbruch in der Region der Malwinen stattgefunden habe und der Forderung nach Abzugder Argentinier. Hieraus zog Großbritannien seinerseits die Rechtfertigung "zurückzuschlagen".

$26 \mathrm{~S} / 15048$, S. 2.

27 In: Vereinte Nationen, Bonn, Heft 4/1982, S. 148.

28 In: Vereinte Nationen, Bonn, Heft 4/1982, S. 148. 
ßere Zahl, insbesondere der Commenwealth-Länder, ${ }^{29}$ das britische Vorgehen als Verteidigung gegen eine Aggression rechtfertigte, nahm auch bei anderen Staaten, die den argentinischen Einmarsch mißbilligt hatten, die Kritik an der britischen Politik der Gewaltanwendung zu. So schrieb der kolumbianische Präsident Turbay-Ayala, dessen Regierung noch am 28. 4. 1982 auf einer Tagung des Rio-Paktes einer Resolution nicht zustimmte, die Argentinien unterstützte, ${ }^{30}$ am 21 . Mai einen Brief an die britische Regierung, in dem zur Zurückhaltung aufgefordert wird:

"Ich weiß, daß die ersten militärischen Aktionen zwischen Argentiniern und Briten stattgefunden haben, und sehe mit Schrecken die Ausweitung des Krieges mit all seinen verhängnisvollen Konsequenzen. Meine Regierung ist der Auffassung, daß die demokratischen Nationen die Verpflichtung haben, das System gegen die es bedrohenden Risiken zu stärken. Nach unserer Auffassung kann dieses Ziel nicht erreicht werden, wenn wir uns selbst in blutigen und unfruchtbaren Kriegen schwächen, sondern nur, wenn wir uns mit der notwendigen Geduld bewaffnen und an einem Dialog teilnehmen, bis ein Punkt der Ubereinstimmung gefunden werden kann, der die Zuversicht der Welt erneuert, die Gefahren eines neuen Kriegsbrandes zu beseitigen. " ${ }^{31}$

Daß die englische Interpretation, nach der mit der Resolution 502 (1982) eine Zustimmung zu militärischen Aktionen enthielt, auf immer stärkere Kritik stieß, machten die Auseinandersetzungen im Sicherheitsrat Anfang Juni deutlich, als ein von Spanien und Panama eingebrachter Antrag auf sofortigen Waffenstillstand ${ }^{32}$ nur am Veto der USA und Großbritanniens scheiterte. ${ }^{33}$ "Großbritannien lehnte den Entwurf mit der Begründung ab, ein Waffenstillstand ohne sofortigen und bedingungslosen Abzug der Argentinier sei nicht akzeptabel. ${ }^{34}$

Die Differenzen unter den Blockfreien zeigten sich im Abstimmungsverhalten. Panama, Uganda und Zaire stimmten für diese Resolution, während Guavana, Togo und Jordanien sich der Stimme enthielten. Zur Begründung erklärte der Vertreter Zaires seine Zustimmung mit der Notwendigkeit, die Resolutionen 502 (1982) und 505 (1982) zu verwirklichen..$^{35}$ Guayana bemängelte, daß in der Resolution keine Beziehung zwischen der Aufforderung nach einem Waffenstillstand und der Verpflichtung Argentiniens hergestellt wurde, "seine Truppen innerhalb eines klar definierten Zeitplanes von den Falklands zurückzuziehen. ${ }^{36}$

29 Nach einer Meldung des Guardian vom 3. 11. 1982 erklärten 39 der 47 Commonwealth-Staaten ihre Zusamming zu solchen Aktionen.

30 Der Fischer Welt Almanach '83, Frankfurt 1982, S. 93.

$31 \mathrm{~S} / 15126, \mathrm{~S} .1 \mathrm{f}$.

32 S/15156/Rev. 2, in: Vereinte Nationen, Bonn, Heft 4/1982, S. 148.

33 Wenige Minuten nach der Abstimmung erklärte die amerikanische UN-Delegierte J. Kirkpatrick, daß das Veto der USA auf einen Ubermittlungsfehler zurückzuführen ist. Wenn möglich, würde sie das amerikanische Abstimmungsverhalten in Enthaltung verändern.

34 Dokumente zum Konflikt um die Falkland-Inseln, in: Europa-Archiv, 37. Jg., 19. Folge, 1982, D 475.

35 UN-Chronicle, July 1982, Vol. XIX, No. 7., S. 18.

36 Security Council, Provisional Verbatim Record, S/PV. 2373, S. 17.

37 Süddeutsche Zeitung, 6. Juni 1982. 


\section{Die Ministerkonferenz des Koordianationsbüros in Havanna}

Die sich so herausbildenden Widersprüche kamen auf der Ministertagung des Koordinationsbüros, das vom 31. 5.-5. 6. 1982 in Havanna tagte, voll zum Durchbruch. Obwohl diese Konferenz eigentlich der Vorbereitung der für Anfang September 1982 in Bagdad geplanten Gipfelkonferenz dienen sollte, nahmen die Ereignisse im südlichen Atlantik einen großen Stellenwert ein. Angesichts der aktuellen Zuspitzung des Konflikts und der Tatsache, daß die Konferenz in Lateinamerika stattfand, kann dies nicht verwundern.

Auch Argentinien maß dieser Konferenz große Bedeutung bei und entsandte nach neun Jahren zum ersten Mal wieder einen Außenminister (Costa Mendez) zu einer blockfreien Konferenz. Die alten Widersprüche mit Kuba schienen sich in Luft aufgelöst zu haben. Fidel Castro ließ den argentinischen Gästen eine Sonderbehandlung zukommen. Auch Costa Mendez fand "lobende Worte für jene Staaten, die sich aus der Abhängigkeit von den Großmächten befreit hätten. Als Beispiele ... nannte der Minister Algerien, Indien, Cuba - 'die Perle der Karibikı - und Vietnam. ${ }^{37}$ Ein Handels- und Wissenschaftsabkommen zwischen beiden Ländern im Wert von rund 230 Millionen Mark bekräftige diese neue Freundschaft.

Wie zu erwarten stand auf der Konferenz selbst die Frage nach einer Verurteilung Großbritanniens und der USA im Zentrum der Auseinandersetzung. War die britische Rückeroberung der Inseln durch die UN-Charta und die Sicherheitsratsresolutionen gedeckt oder nicht ? $^{38}$ Das verabschiedete Abschlußdokument nimmt deutlicher als die Erklärung des Koordinationsbüros vom 26. April und 5. Mai zugunsten Argentiniens Stellung, enthält aber auch offen widersprüchliche Positionen.

Die bekannten Auffassungen der Blockfreien werden an vielen Punkten bekräftigt, einschließlich der Forderungen nach friedlicher Lösung von Streitfragen. Im konkreten stehen sich aber die Unterstützung der Resolution 502 (1982) und die Aussage gegenüber, daß "die Minister . . . ihre feste Solidarität mit Argentiniens Anstrengungen (bekräftigen), die anachronistische koloniale Situation auf den Malwinen zu beenden und ihre Wiedererrichtung zu verhindern. ${ }^{39}$ Da gleichzeitig die zunehmenden "Spannungen ... (im) Südatlantik ..., insbesondere als Ergebnis der imperialistischen und kolonialisti-

38 Auch H. Weber kommt zu einem ablehnenden Ergebnis: Recht und Gewalt im Südatlantik, in: Vereinte Nationen, Bonn, Heft 3/1982, S. 77-82. Luis Quinteros-Yanez kommt zu dem Ergebnis, daß es sich um eine britische Aggression handeln würde. Der Konflikt zwischen Argentinien und Großbritannien um die Malwinen (Falkland)-Inseln. - Eine Fallstudie zu einigen Aspekten der faktischen und rechtlichen Lage. In: O. Bayer/ M. Ehrke/L. Quinteros-Yanez, Uberlegungen zum Falkland-Malwinen-Konflikt, Institut für Ibero-Amerika-Kunde, Arbeitsunterlagen und Diskussionsbeiträge No. 16, Hamburg, Juli 1982.

39 Der Malwinen-Frage sind die Punkte 108-117 der politischen Deklaration gewidmet, sowie der Punkt 41 der Okonomischen Deklaration. Der vollständige Text der Dokumente befindet sich in: Ministerial Meeting of the Coordinating Bureau of the Nonaligned Countries, Final Communique, Havanna, 31. Mai-5. Juni, S. 27 f. und S. 51. Die in der Dokumentation des Europa-Archivs abgedruckte Fassung stimmt in Numerierung und Inhalt nicht ganz mit der Abschlußresolution überein. Es handelt sich dabei vermutlich um den Text des von Kuba vorgelegten Resolutionsentwurfs.

Die zitierte Stelle stammt aus Punkt 110 der Schlußdeklaration. Hervorhebung durch KF. 
schen Aggressions- und Interventionspolitik ${ }^{40}$ dargestellt werden, und nur die "militärischen Operationen" bedauert werden, die "mit einem großen militärischen Aufgebot des Vereinigten Königreichs und mit Unterstützung und Hilfeleistung der Vereinigten Staaten durchgeführt werden ", ${ }^{41}$ ist die argentinische Freude über diese Resolution mehr als verständlich.

Die Unzufriedenheit einer großen Zahl von Blockfreien ist unter diesen Umständen nicht verwunderlich, was sich in einer großen Zahl von Vorbehalten gegen diese Paragraphen niederschlägt, ${ }^{42}$ die nicht nur von den Mitgliedern des Commenwealth unter den Blockfreien formuliert wurde. In den Vorbehalten wird u. a. kritisiert, daß die verabschiedeten Paragraphen "nicht die grundlegenden Tatsachen und die Realität der Situation widerspiegeln" (Kenia). ${ }^{43}$ Von Seiten Botswanas wurde erklärt, daß Großbritannien keine Aggression begangen habe, und die ghanesische Regierung wies darauf hin, daß "der Vorbehalt auf der Tatsache basiert, daß Ghanas Ausdruck der Solidarität mit . . . Argentinien selbst das blockfreie Prinzip der Nichtanwendung von Gewalt in der Lösung von Streitfragen zum Gegenstand machen muß." ${ }^{44}$

Diese Widersprüchlichkeit der Resolution macht zum einen die tiefen Widersprüche deutlich, die innerhalb der Blockfreien existierten und nicht in einer gemeinsamen, konsistenten Resolution, aufgelöst werden konnten. Aber sicherlich hat auch das gewählte Entscheidungsverfahren zu diesem Ergebnis beigetragen. In einem Vorbehalt bedauert die Vertretung Singapurs, "daß die nicht-lateinamerikanischen Delegationen nicht die adäquate Möglichkeit bekamen, aktiv bei der Formulierung der Paragraphen mitzuwirken." ${ }^{45}$ Damit wird kritisiert, daß es zunehmend Brauch wird, die Paragraphen zu einzelnen Regionen von den zuständigen Ländergruppen formulieren zu lassen. Dies muß in dem Moment zu einem Dissens führen, in dem eine dieser Gruppe eine relativ geschlossene Position vertritt (wie hier Lateinamerika), die von den anderen Regionalgruppen nicht geteilt wird.

40 Punkt 109 der Schlußresolution.

41 Punkt 114 der Schlußresolution.

42 Da es beider Blockfreienbewegung keine Abstimmung gibt, ist der Vorbehalt die Methode, abweichende Auffassungen im und zum verabschiedeten Dokument zum Ausdruck zu bringen. Vgl. dazu den Anhang I der Schlußdeklaration der Gipfelkonferenz von Havanna, in: Internationale Politik (Belgrad), No. 707, S. $68 \mathrm{f}$. Auf der Konferenz von Havanna formulierten zu den Malwinen-Paragraphen folgende Länder einen Vorbehalt: Kamerun, Botswana, Sierra Leone, Zentralafrikanische Republik, Lesotho, Sudan, Swaziland, Zaire, Ghana, Singapur, Bangladesh, Bhutan, Saudi-Arabien, Jordanien, Oman, Bahrein, Jamaica. Obwohl sich diese Vorbehalte auf verschiedene Paragraphen verteilen (die höchste Zahl von Vorbehalten wurde mit 12 gegen den Paragraphen 112 eingelegt), ist diese Zahl sehr hoch, wenn man bedenkt, daß sicherlich nicht alle Vorbehalte auch als solche kenntlich gemacht worden sind.

Die gesamten Vorbehalte sind wiedergegeben, sof ern sie schriftlich begründet wurden, im Anhang des Schlußkommuniques der Ministerkonferenz des Koordinationsbüros in Havanna, a.a.O., S. 145 -". 201.

43 Ministerial Meeting of the Coordinating Bureau of the nonaligned countries, Havanna, a.a.O., S. S. 168.

44 Ebenda, S. 158.

45 A.a.O., S. 183. 


\section{Für die Stärkung der Rolle der Vereinten Nationen}

Die Blockfreien beschränkten sich jedoch nicht auf Stellungnahmen zu diesem Kriegsfall. Um eine friedliche Lösung des Konfliktes zu erreichen, setzten sich für die Stärkung des Systems der Vereinten Nationen ein. Sie selbst unternahmen keine eigenen Friedens- oder Vermittlungsmissionen. Zentrale Bedeutung sollte, in Verwirklichung der UN-Charta, dem UN-Generalsekretär Perez de Cuellar zukommen. ${ }^{46}$ Nach informellen Vorbereitungen, und nachdem bis zum 27. April die Hauptanstrengungen in diese Richtung vom amerikanischen Außenminister Haig durchgeführt worden sind, enthält die Erklärung des Präsidenten des Sicherheitsrates vom 5. Mai 1982 einen solchen Auftrag. ${ }^{47}$

Da die Blockfreien besorgt waren, daß der Sicherheitsrat nicht genügend Aktivität zeigte, diskutierten den April 1982 hindurch meinige lateinamerikanische blockfreie Länder die Idee einer blockfreien Initiative zur Lösung des Falklandkonflikts durch eine aktivere Einbeziehung der Vereinten Nationen. Diese Länder gingen davon aus, daß die gegenwärtige Krise die Frage der Dekolonisierung einbezieht, und als solche, die Vereinten Nationen die Verantwortung tragen zu intervenieren und eine friedliche Lösung des Problems auszuarbeiten.

Eine Mehrheit blockfreier Länder, die sich der Wiederbesetzung der Inseln durch Großbritannien widersetzten, argumentierten, daß, wenn Argentiniens Militäraktion zur Inbesitznahme der Falklands ungerechtfertigt war, dann war Großbritannien noch weniger berechtigt, sie zurückzuerobern. Es wurde deshalb durch diese Länder angeregt, daß unter solchen Umständen die Falklandinseln den Vereinten Nationen zur Treuhandschaft übergeben werden sollten und daß ihre Zukunft bestimmt werden könnte in Úbereinstimmung mit den üblichen Dekolonisierungsprozeduren. ${ }^{48}$

Solche Vorstellungen spielten dann auch in den Vorschlägen, die der UN-Generalsekretär den Konflikparteien Argentinien und Großbritannien unterbreitete, eine wichtige Rolle. In seiner Erklärung vor dem Sicherheitsrat am 21. Mai 1982 erklärte er, daß bei Fortbestand weiterer wichtiger Differenzen "ein wesentliches Einverständnis" u. a. über die folgenden Punkte erzielt worden sei:

"4. Der Truppenabzug würde . . . unter Kontrolle von Beobachtern der Vereinten Nationen stehen.

5. Die Ubergangsverwaltung des Gebiets stünde unter der Autorität und der Flagge der Vereinten Nationen. Argentinien und das Vereinigte Königreich würden kleine Verbindungsbüros unterhalten, auf denen ihre jeweilige Flagge wehen könnte.

Perez de Cuellar kommt aus einem blockfreien Land und wurde mit Unterstützung der Blockfreien zum Generalsekretär gewählt.

47 Vereinte Nationen, Bonn, Heft 4/1982, S. 148.

48 Madan Lal, The Falkland Crisis: A Preliminary Assessment, in: Foreign Affairs Reports, Vol. XXXI, No. 6, June 1982, S. 111. Im gleichen Auf satz befindet sich auch eine kurze Charakterisierung der indischen Position. 
6. Die Seiten würden in redlicher Absicht unter der Ägide des Generalsekretärs der Vereinten Nationen in Verhandlungen zur friedlichen Lösung des Streits eintreten ... ${ }^{49}$

Entsprechend dieser Vorschläge ermächtigte der Sicherheitsrat den UN-Generalsekretär in der Resolution 505 (1982), einen Waffenstillstand auszuhandeln, der "erforderlichenfalls auch ... Vereinbarungen über die Entsendung von Beobachtern der Vereinten Nationen zur Überwachung der Einhaltung der Waffenstillstandsbedingungen "50 enthält.

Diese Vorstellungen fanden sowohl die Zustimmung der blockfreien Mitglieder des Sicherheitsrates, wie dem Ministertreffen des Koordinationsbüros in Havanna, das seine "Unterstützung für die Anstrengungen" des UN-Generalsekretärs erklärte. ${ }^{51}$

\section{Die Malwinen-Frage auf der 37. Generalversammlung der UN (1982)}

Bis zum Abschluß der Feindseligkeiten durch die britische Rückeroberung der Malwinen waren weder der Generalsekretär, noch der Sicherheitsrat in der Lage, mäßigend auf die Konfliktparteien einzuwirken. Der Konflikt entwickelte sich "weitgehend an diesem Hauptorgan der Vereinten Nationen vorbei“. ${ }^{52}$ Entsprechendes gilt natürlich auch für die Blockfreienbewegung.

Seitdem versucht Argentinien wieder, mit Unterstützung der anderen lateinamerikanischen Staaten, das Form der Vereinten Nationen für die Diskussion dieser Frage zu nutzen. So forderten die Außenminister von 20 lateinamerikanischen Ländern die Aufnahme des Tagesordnungspunktes "Die Frage der Malwinen" in die Tagesordnung der 37. Generalversammlung. ${ }^{53}$ Dort fand vom 2.-6. November eine entsprechende Debatte statt, erstmals als eigener Tagesordnungspunkt.

Für diese Debatte war von den gleichen lateinamerikanischen Ländern ein Resolutionsentwurf eingebracht worden. Die nach viermaligen Veränderungen - unter Mitarbeit der USA - verabschiedete Resolution war äußerst moderat. Sie verweist darauf, daß "koloniale Situationen mit dem universellen Friedensideal . . unvereinbar" sind, ${ }^{55}$ verweist auf die Resolutionen der Generalversammlung und des Sicherheitsrates, stellt eine "defacto-Einstellung" der Feindseligkeiten fest, bekräftigt die Notwendigkeit, "den Interessen der Bevölkerung der Falkland-Inseln (Malwinen) gebührend Rechnung zu tragen",

49 Erklärung des Generalsekretärs der Vereinten Nationen vor dem Sicherheitsrat am 21. Mai 1982, in: Dokumente ..., Europa-Archiv, a.a.O., D 498 (1).

50 In: Vereinte Nationen, Bonn, Heft 4/1982, S. 148.

51 Punkt 116 der Schlußdeklaration der Ministerkonferenz des Koordinationsbüros in Havanna, a.a.O., S. 28.

52 Hermann Weber, Falkland-Inseln, in: Vereinte Nationen, Bonn, "Aus dem Bereich der Vereinten Nationen", Heft 5/1982, S. 172.

53 A/37/193. Unter den Unterzeichnern waren aus der Karibik nur die Dominikanische Republik, Haiti und Kuba, während vom lateinamerikanischen Festland Belize, Guavana und Suriname fehlten.

54 Entfällt.

55 A/RES/37/9 vom 4. November 1982, zit. nach der inoffiziellen deutschsprachigen Vorauskopie. Protokolle der Diskussion in A/37/PV. 51-56. 
bekräftigt - die Grundsätze der Charta der Vereinten Nationen und ersucht Argentinien und Großbritannien Verhandlungen durchzuführen, um zu einer "friedlichen Lösung des . . S Souveränitätskonfliktes zu gelangen“. Darüberhinaus wird der Generalsekretär gebeten, "erneut seine guten Dienste zur Verfügung zu stellen". Die Resolution verzichtet aber auf jeden Hinweis auf die Beschlüsse der Blockfreienbewegung und der amerikanischen Staaten, die das Recht der Republik Argentinien auf die Malwinen unterstützen.

Da England zur Zeit jedoch jegliche Verhandlung über die Malwinen ablehnt, kam es hinter den Kulissen zu einem heftigen Tauziehen. Großbritannien hoffte, daß die relativ große Unterstützung, die es während des Krieges erhalten hatte, sich auch in einer Ablehnung dieser Resolution niederschlagen würde, zumindest aber in einem ungefähren Pari. Diese Rechnung ging aber nicht auf. So stimmte zum einen die USA trotz eines Protestens von M. Thatcher an den amerikanischen Präsidenten Reagan für die Resolution, um die bevorstehende Lateinamerikareise Reagans nicht zu sehr zu belasten. Neben Großbritannien stimmten nur 11 Länder gegen die Forderung nach Verhandlungen (darunter 5 blockfreie). 90 stimmten der Resolution zu (darunter 60 Blockfreie), 52 Länder enthielten sich (darunter 30 blockfreie). Diese Enthaltungen gaben dabei dem Wunsch nach baldiger Wiederaufnahme der Verhandlungen Ausdruck, wollten aber entweder erneut ihrer Kritik an Argentinien Ausdruck geben oder deutlich machen, daß die Rolle des Selbstbestimmungsrechtes der Bewohner der Malwinen in erster Linie zu entsprechen ist. ${ }^{56}$ So wurde diese Abstimmung in Großbritannien zu Recht als Niederlage empfunden, da die große Mehrheit ihrem Wunsch nach baldigen Verhandlungen - wenn auch in unterschiedlicher Weise - Ausdruck gab.

\section{Verstärkung blockf reier Tendenzen in Lateinamerika}

Fast ein Jahr nach Ausbruch des Konfliktes ist deutlich geworden, daß diese Auseinandersetzung um die "hergebrachte Weltordnung “57 Folgen hatte, die weit über das bilaterale Verhältnis der beiden Konfliktparteien hinausgehen. Maßgeblich dafür ist vor allem, daß die USA die "amerikanische Einheit" zugunsten ihres europäischen Verbündeten gesprengt hat. Als Folge traten die bis dahin existierenden Konflikte in Lateinamerika selbst in den Hintergrund. Diese Annäherung schloß auch Kuba und Nicaragua mit ein, die man lange Zeit versuchte zu isolieren. Und dieser Prozeß ist nicht auf Argentinien beschränkt, sondern umfaßt nahezu alle lateinamerikanischen Länder. Ein Ausdruck davon ist die zunehmende Diskussion über die Bildung eines eigenen lateinamerikanischen politischen Zusammenschlusses, da die Organisation Amerikanischer Staaten (OAS) immer weniger den Bedürfnissen Lateinamerikas entspreche. Insgesamt kann

56 So z. B. Antigua, Fidschi, Zaire, Jamaica, Bahamas, Senegal, Trinidad und Tobago, Guayana und Sierra Leone.

57 So Wolfgang Wagner, Der Konflikt um die Falkland-Inseln. Ein Streit um die hergebrachte Weltordnung, in: Europa-Archiv, 37. Jg. Folge 17, S. 509-516. 
man sagen, daß eine stärkere Orientierung Lateinamerikas zur Dritten Welt und der Blockfreienbewegung deutlich zu beobachten ist.

So wird die Umorientierung Argentiniens in Richtung Blockfreienbewegung, die sich schon auf der Konferenz in Havanna andeutete, umso deutlicher, wenn man sich vor Augen führt, daß der argentinische Außenminister Costa-Mendez, der auch Kuba besuchte, noch im Frühjahr 1982 "bei einem Besuch in Brasilien die Auffassung geäußert (hatte), daß Argentinien nicht als Teil der Dritten Welt betrachtet werden könne ... Und im November vorigen Jahres (also 1981, KF) hatte . . General Galtieri bei einem Besuch in Washington erklärt, Argentinien und die USA marschierten gemeinsam in dem rideologischen Krieg, der auf der Welt geführt wird. ${ }^{58}$

Diese Stimmung hat auch nach dem Ende der offenen Feindseligkeiten nicht nachgelassen. Im Gegenteil geht heute "die Stimmung allgemein dahin, die argentinische Außenpolitik auf der lateinamerikanischen Achse in der Nähe der Dritten Welt anzusiedeln; nur aus Gründen der Notwendigkeit und Zweckmäßigkeit soll sie bezüglich Technologie und Handel gegenüber Europa und den Vereinigten Staaten offenbleiben. ${ }^{59}$ Die Folgen sind, wenn auch noch nicht in allen Aspekten eindeutig und unumstritten, offensichtlich. So wird Argentinien auf der 7. Gipfelkonferenz der Blockfreien mit dem Staatschef vertreten sein. Ein weiteres Indiz ist die Auseinandersetzung in der argentinischen Armee über das Verhältnis zu Südafrika. Nachdem der argentinische Stabschef erklärte, daß "Argentinien und Südafrika gleiche Freunde" hätten und gegen die "gleichen Feinde" kämpften, wurde dies offiziell zurückgewiesen. "Argentinien . . habe in den letzten Jahren immer eindeutig zu verstehen gegeben, daß es vor allem mit der Apartheidpolitik der südafrikanischen Regierung nicht übereinstimme. " ${ }^{60}$

Auch andere Länder suchen zunehmend einen stärkeren Kontakt zu den Blockfreien. Nach Ekuador, das bereits seit September 1981 Vollmitglied ist, ${ }^{61}$ wurden auf der 7. Gipfelkonferenz in Neu Delhi (März 1983) die Bahamas, Barbados und Kolumbien neue Vollmitglieder. ${ }^{62}$ Die damit verbundene Annäherung an Kuba und Nicaragua ${ }^{63}$ ist unübersehbar. In diese Reihe von Ereignissen paßt auch, daß Bolivien anläßlich der Ministertagung des Koordinationsbüros in Managua (10.-14. Januar 1983) seine diplomatischen Beziehungen zu Kuba wieder aufgenommen hat. ${ }^{64}$

\section{Einige Schlußfolgerungen}

Betrachtet man die Auswirkungen des Krieges um die Malwinen auf die Blockfreienbewegung, so sind nach dem bisher gesagten zwei deutliche Tendenzen erkennbar. Zum ei-

58 Hamburger Abendblatt, 4. Juni 1982.

59 Mariano C. Grondona, Argentinien: Ein tiefer Einschnitt. In: Europa-Archiv, 37. Jg., Folge 23, S. 701.

60 Frankfurter Allgemeine Zeitung, 29. 12. 1982.

61 Entfällt.

62 Venezuela zog seinen Antrag wegen des Widerstands Guayanas zurück.

63 Martin Gester, Kolumbien mit neuer Außenpolitik, Frankfurter Allgemeine Zeitung, 25. 1. 1983.

64 Kommunique in Granma Weekly, Havanna, 18. Jg., No. 4, 23. 1. 83. 
nen die stärkere Hinwendung Lateinamerikas zu den Blockfreien als Ausdruck der Enttäuschung über das "inneramerikanische Sicherheitssystem". "Die Krisen um die Falklandinseln bestritt of fensichtlich viele Grundlagen dieses Systems und eröffnete den Prozeß der Errichtung neuer inneramerikanischer Beziehungen, in welchen die blockfreien Solidarität der Länder Lateinamerikas über die blockmässigen, in den Verhältnissen des kalten Krieges und der totalen wirtschaftlichen Abhängigkeit dieses Kontinents von der Wirtschaft der USA entstandenen Bindungen die Oberhand gewinnen wird. " ${ }^{65}$

Auf der anderen Seite hat die Auseinandersetzung nicht nur unterschiedliche Standpunkte über die Gewaltanwendung Argentiniens zum Ausdruck gebracht - wobei diese von der Mehrheit nicht gebilligt wurde -, sondern of fensichtlich auch den lange vorhandenen Konsens über die Unterstützung des argentinischen Anspruchs auf die Malwinen gelockert. Der Krieg um die Malwinen hat dazu geführt, daß der Konflikt um ein unbedeutendes Stück Land, weit entfernt von Asien und Afrika, an Bedeutung für das internationale politische System gewann. Auch ist der Druck von Seiten Großbritanniens größer geworden, das jetzt von seiner lange Zeit erklärten Bereitschaft einer Souveränitätslösung nichts mehr wissen will. So hat die Zahl der Länder, die sich dem Souveränitätsanspruch Argentiniens öffentlich widersetzen, in den letzten Jahren zugenommen. Weiterhin, so hat das Verhalten der Blockfreienbewegung als Ganzes und der einzelnen Länder in ihrer Mehrheit deutlich gemacht, ist einer Einschätzung nicht zuzustimmen, daß im Konflikt um die Malwinen die "große Mehrheit der Staaten", "vor ein konkretes Problem der Weltordnung gestellt", "dem dynamischen Prinzip der Auflösung einer überkommenen rechtlich gesicherten Ordnung den Vorzug vor dem statischen Grundsatz der Wahrung des Rechts (gaben). Die Hüter des Rechts innerhalb der Staatengesellschaft mußten erkennen, daß sie nur noch eine Minderheit darstellen. "66

Mit der Entwicklung des Konflikts kam bei den Blockfreien eine differenziertere Position zum Ausdruck. Nach der mehrheitlichen Ablehnung der argentinischen Gewaltanwendung, wurde auch das völkerrechtswidrige Vorgehen Großbritanniens verurteilt. Stattdessen setzten sich die Blockfreien dafür ein, den Konflikt auf friedlichem Wege mit Hilfe der Vereinten Nationen zu lösen. Daß diese Bemühungen erfolglos blieben, dafür trägt insbesondere Großbritannien die Verantwortung, das auf die völkerrechtswidrige Inbesitznahme der Inseln durch Argentinien mit einer Demonstration der Stärke, $10000 \mathrm{~km}$ von London entfernt, seinen kolonialen Besitz mit Gewalt verteidigte. 


\title{
The Non-Aligned Movement in the Falkland Conflict
}

\author{
by Klaus Fritsche
}

The Malvinas (Falklands) war not only intensified tensions between Great Britain and Argentina, but also led Latin American countries to identify themselves increasingly with the Third World and the Non-Aligned Movement.

Amongst other facts this is due to the support, which the Non-Aligned Movement has given for Argentina's right to the restitution of the Malvinas (Falklands) and sovereignty over them since the Foreign Minister Conference of Non-Aligned Countries in Lima (1975). At the beginning of the war between Great Britain and Argentina (April 1982) the majority of non-aligned states reiterated their support for Argentina's claim but refused to support Argentina's use of force. They voted for a peaceful solution and tried to bring the conflict to an end under the assistance of the United Nations. Therefore the majority of member countries also criticised Britain's military actions.

The Malvinas (Falklands) war also led to further differences of opinion within the NonAligned Movement itself. The number of members, predominantly belonging to the Commonwealth of Nations, which refused to accept Argentina's claim, has grown. A great number of member states also supported Britain's military actions.

Therefore one of the consequences of the war was the weakening of the unity of the NonAligned Movement. Nevertheless, the 37th General Assembly of the United Nations showed the will of the overwhelming majority of non-aligned states to continue their work for a peaceful solution of the conflict. 